\title{
Salivary enzymatic antioxidant activity and dental caries: A cross-sectional study
}

\author{
Zakaria Vahabzadeh ${ }^{1,2, A-F}$, Zahra Moaven Hashemi ${ }^{3, B}$, Bijan Nouri ${ }^{4, C}$, Fatemeh Zamani ${ }^{5, B}$, Faranak Shafiee ${ }^{6, A-F}$ \\ ${ }^{1}$ Liver and Digestive Research Center, Research Institute for Health Development, Kurdistan University of Medical Sciences, Sanandaj, Iran \\ ${ }^{2}$ Department of Biochemistry, Faculty of Medicine, Kurdistan University of Medical Sciences, Sanandaj, Iran \\ ${ }^{3}$ Student Research Committee, Kurdistan University of Medical Sciences, Sanandaj, Iran \\ ${ }^{4}$ Social Determinants of Health Research Center, Research Institute for Health Development, Kurdistan University of Medical Sciences, Sanandaj, Iran \\ ${ }^{5}$ Cellular and Molecular Research Center, Research Institute for Health Development, Kurdistan University of Medical Sciences, Sanandaj, Iran \\ ${ }^{6}$ Department of Pediatric Dentistry, Faculty of Dentistry, Kurdistan University of Medical Sciences, Sanandaj, Iran
}

A - research concept and design; $\mathrm{B}$ - collection and/or assembly of data; $\mathrm{C}$ - data analysis and interpretation;

$D$ - writing the article; $E$ - critical revision of the article; $F$ - final approval of the article

\section{Address for correspondence}

Faranak Shafiee

E-mail: faranakshafiee@gmail.com

\section{Funding sources}

This work, as a dentistry student's thesis, was supported financially by the Vice-Chancellor for Research of Kurdistan University of Medical Sciences in Sanandaj, Iran (grant No. IR.MUK.REC.1397/230).

\section{Conflict of interest}

None declared

\section{Acknowledgements}

We would like to thank the Heads of the Cellular and Molecular Research Center of Kurdistan University of Medical Sciences in Sanandaj, Iran, for their valuable assistance.

Received on June 7, 2020

Reviewed on July 5,2020

Accepted on August 6, 2020

Published online on December 31, 2020

Cite as

Vahabzadeh Z, Hashemi ZM, Nouri B, Zamani F, Shafiee F.

Salivary enzymatic antioxidant activity and dental caries: A cross-sectional study. Dent Med Probl. 2020;57(4):385-391. doi:10.17219/dmp/126179

DOI

$10.17219 / \mathrm{dmp} / 126179$

Copyright

○) 2020 by Wroclaw Medical University

This is an article distributed under the terms of the

Creative Commons Attribution 3.0 Unported License (CC BY 3.0)

(https://creativecommons.org/licenses/by/3.0/).

\begin{abstract}
Background. Oxidative stress has been identified as a predisposing factor for dental caries. Saliva, as a rich source of antioxidants, plays an essential role in the protection against dental caries. Salivary enzymatic antioxidants include superoxide dismutase (SOD), catalase (CAT) and glutathione peroxidase (GPX).

Objectives. The aim of this study was to evaluate the correlation of salivary enzymatic antioxidant activity with different levels of dental caries in children.

Material and methods. In this cross-sectional study, 90 healthy children aged 7-12 years (36 girls, 54 boys) were investigated. Demographic information was gathered and dental examinations were provided for all participants. Then, unstimulated whole saliva samples were collected in the morning. The salivary SOD, CAT and GPx activity was measured spectrophotometrically. For statistical analysis, Spearman's correlation test, the Mann-Whitney U test and the Kruskal-Wallis test were used with the SPSS for Windows software, v. 16.

Results. Our results showed no significant correlation between SOD, CAT and GPx and the decayed, missing, filled teeth index for permanent/primary dentition (DMFT/dmft). The CAT activity was elevated in proportion to the number of decayed teeth. The SOD activity showed a positive correlation with the frequency of tooth brushing. The activity of SOD, CAT and GPx was higher in boys than in girls. An inverse relationship between enzymatic antioxidant activity and age was also observed.
\end{abstract}

Conclusions. Although enzymatic antioxidants had no positive correlation with DMFT/dmft, they were positively correlated with the number of decayed teeth and the improvement of oral hygiene.

Key words: dental caries, saliva, oral hygiene, enzymatic antioxidant 


\section{Introduction}

Dental caries is a dynamic and continuous process. It involves repeated cycles of demineralization by the acidic metabolites produced through the fermentation process of dietary carbohydrates of microbial origin, followed by remineralization by salivary components. Bacterial infection is one of the main etiologic factors for dental caries. The substrate and the host are also crucial factors for the initiation of the caries process. Clinical studies have shown that saliva, as a host factor, plays an essential role in the progression or regression of oral diseases, and in some cases, it regulates them. It is well established that the salivary protective mechanisms control the process of dental caries to a great extent. ${ }^{1}$ Saliva is a heterogeneous liquid and a multifunctional natural defense system. It is a rich source of antioxidants, which play an important role the protection against bacteria, viruses and fungi, also with regard to the mucosa and tooth surfaces, ${ }^{2,3}$

Several studies have been done to identify the components of the natural defense system for the teeth and the oral mucosa. ${ }^{4-6}$ Oxidative stress occurs as a result of an imbalance between reactive oxygen species (ROS) and the antioxidant system. It has been identified as one of the essential predisposing factors for many oral inflammatory conditions. ${ }^{6,7}$ Oxidative stress directly affects the underlying mechanisms which induce the intracellular signals leading to the growth and differentiation of cariogenic bacteria. ${ }^{8}$ Recent studies provided documentation indicating the elevation of oxidative damage biomarkers in the saliva of patients with periodontal diseases and dental caries, ${ }^{2}$ but it is not yet known whether the elevation of these biomarkers is due to the increased production of ROS or antioxidant deficiency. Interventional studies on humans and animal models showed the beneficial effect of supplementation with antioxidants to prevent or reduce the progression of periodontitis., ${ }^{3,5}$,

The salivary antioxidant system contains both enzymatic and non-enzymatic antioxidants. The enzymatic antioxidant defense system includes superoxide dismutase (SOD), catalase (CAT) and glutathione peroxidase (GPx). ${ }^{10}$ Enzymatic antioxidants make up the first line of antioxidant defense. They inhibit the release of primary free radicals and other following chemical chain reactions. $^{10}$

Superoxide dismutase is an antioxidant enzyme that catalyzes 2 superoxide anion molecules to hydrogen peroxide $\left(\mathrm{H}_{2} \mathrm{O}_{2}\right)$ and oxygen $\left(\mathrm{O}_{2}\right)$ molecules. ${ }^{10}$ This enzyme is essential for cellular health and body protection against free radicals. The SOD levels decrease with age. A daily intake of SOD supplements has been shown to improve the immune system potential and reduce the chances of disease. ${ }^{10}$

Glutathione peroxidase is another enzymatic antioxidant that plays an essential role in neutralizing $\mathrm{H}_{2} \mathrm{O}_{2}$ produced through the SOD dismutation process. ${ }^{11}$
It is mainly found in mitochondria, and sometimes in cytosol. Glutathione peroxidase breaks down $\mathrm{H}_{2} \mathrm{O}_{2}$ into water as well as lipid peroxides into their relevant alcohols. ${ }^{12}$ It also plays an important role in the prevention of cancer and cardiovascular diseases. ${ }^{10}$

Catalase is a ubiquitous antioxidant enzyme, found in almost all living organisms using oxygen. It has a secondary role in defense against peroxidative agents. ${ }^{13}$ This enzyme catalyzes $\mathrm{H}_{2} \mathrm{O}_{2}$ produced through the dismutation process to water and $\mathrm{O}_{2}$ molecules, thus completing the detoxification process after SOD. Patients with CAT deficiency (acatalasemia) are more likely to develop type 2 diabetes mellitus. ${ }^{10}$

It has been shown that imbalances in the SOD, CAT and GPx ratios can lead to disease. ${ }^{13-15}$ Hendi et al. showed decreased SOD activity and increased CAT and GPx activity in children with dental caries as compared to those without caries. ${ }^{4}$ It has been suggested in recent years that salivary oxidative stress markers could be used to screen and control oral diseases, including dental caries. ${ }^{3}$ The evaluation of these salivary markers could help identify patients who are reluctant to undergo regular dental examinations or are at high risk for dental caries; additionally, these markers offer useful information concerning the development of new preventive and treatment modalities. ${ }^{3,5,6}$ Also, the availability and non-invasiveness of these techniques are good reason to use saliva as a diagnostic biological fluid to evaluate diseases. ${ }^{2}$ Previous studies showed that the antioxidant status of saliva, especially in children, may reflect the risk for dental caries. ${ }^{16}$ Considering the relationship between antioxidants and the caries process, and the feasibility of the analysis of salivary enzymatic antioxidants, this study aimed to evaluate salivary enzymatic antioxidant levels in children with caries.

\section{Material and methods}

\section{Patient selection}

This cross-sectional study was carried out in 2019 in Sanandaj, Iran. Clinical unstimulated salivary samples were collected from 90 healthy children aged 7-12 years. This age group has more experience of dental caries. ${ }^{17}$ The sample size was determined based on previous studies. ${ }^{2,6}$ Children whose parents received a complete explanation about the aims and methods of the study, and then signed written consent were included in the study. The inclusion criteria of this study were similar to those previously reported by others. ${ }^{2,7}$ Children who had a history of a systemic or local disease affecting salivary secretion and/or received antifungal agents, antihistamines, corticosteroids, nonsteroidal anti-inflammatory drugs (NSAIDs), antimicrobial mouthwashes, or other long-term medications 
or supplements for at least 1 month before the study were excluded. Demographic information was gathered and dental examinations were provided for all participants. The dental caries index was determined according to the World Health Organization (WHO) criteria using a dental mirror and an explorer $24 \mathrm{~h}$ before sampling.

This study was approved by the ethics committee of Kurdistan University of Medical Sciences in Sanandaj, Iran, under IR.MUK.REC.1397/230.

\section{Collection of saliva}

Unstimulated salivary samples were collected between 9 a.m. and 12 a.m., at least $2 \mathrm{~h}$ after the last episode of eating or drinking to reduce any variability resulting from the effect of the circadian rhythm or possible stimulation. Generally, unstimulated saliva contains basal and normal components of saliva that is secreted into the oral cavity during the day. The children were asked to rinse their mouths with distilled water to remove any food debris before the salivary samples were collected. They were also asked to sit quietly on a chair and bend their head slightly downward without any masticating movement to allow saliva to flow from the lower lip into sterile tubes passively. This draining protocol is more reliable and requires no stimulation. ${ }^{18}$ Finally, $5 \mathrm{~mL}$ of saliva was collected in appropriately numbered tubes by means of the abovementioned method. The tubes were then tightly sealed to prevent contamination or evaporation, placed on an ice pack and sent to the laboratory within an hour. The salivary samples were centrifuged at 4,000 rpm for $4 \mathrm{~min}$ at $4^{\circ} \mathrm{C}$. The supernatants were divided in 2 aliquots, and then stored at $-80^{\circ} \mathrm{C}$ until final analysis.

\section{Measurement of enzymatic antioxidant activity}

\section{Superoxide dismutase activity}

The SOD activity of the separated salivary samples was measured using an appropriate assay kit (CAT No. ZB-SOD-96A; ZellBio GmbH, Lonsee, Germany). In brief, in this method, $\mathrm{H}_{2} \mathrm{O}_{2}$ produced during the enzymatic reaction combines with the chromogen component of the kit to provide a complex. Its optical density (OD) was measured in the samples and blank at $420 \mathrm{~nm}$ within 0- and 2-minute time intervals with a microplate reader (Synergy HTX; BioTek Instruments Inc., Winooski, USA). Finally, the SOD activity in the subjects' salivary samples was reported in $\mathrm{U} / \mathrm{mL}$ as mean $(M) \pm$ standard deviation $(S D)$.

\section{Catalase activity}

The CAT activity of the separated salivary samples was measured using an appropriate assay kit
(CAT No. ZB-CAT-96A; ZellBio GmbH). In brief, in this method, a two-step reaction is used to measure the enzymatic activity. In the $1^{\text {st }}$ step, the patients' and blank samples were incubated with a certain amount of $\mathrm{H}_{2} \mathrm{O}_{2}$ for $1 \mathrm{~min}$. In this reaction, $\mathrm{H}_{2} \mathrm{O}_{2}$ dissociates into water and $\mathrm{O}_{2}$. The rate of $\mathrm{H}_{2} \mathrm{O}_{2}$ dissociation was proportional to the CAT concentration in the samples. In the $2^{\text {nd }}$ step, the residual $\mathrm{H}_{2} \mathrm{O}_{2}$ reacts with the existing chromogen to form a complex. Its OD was measured in the samples and blank at $405 \mathrm{~nm}$ after $1 \mathrm{~min}$ with a microplate reader (Synergy HTX; BioTek Instruments Inc.). Finally, the CAT activity in the subjects' salivary samples was reported in $\mathrm{U} / \mathrm{mL}$ as $M \pm S D$.

\section{Glutathione peroxidase activity}

The GPx activity of the separated salivary samples was measured using an appropriate assay kit (CAT No. ZB-GPX-96A; ZellBio GmbH). In brief, in this method, a two-step reaction was used to measure the enzymatic activity. In the $1^{\text {st }}$ step, the samples, control and blank were incubated with a certain amount of glutathione (GSH) for $5 \mathrm{~min}$. In this reaction, GSH is oxidized to glutathione disulfide (GSSG) through the activity of GPx of the samples and control. The amount of the consumed GSH was proportional to the GPx concentration in the samples. In the $2^{\text {nd }}$ step, the residual GSH reacts with the existing chromogen in the kit (Pierce Ellman's Reagent (DTNB); Thermo Fisher Scientific, Waltham, USA) to form a yellow complex. Its OD was measured at $412 \mathrm{~nm}$ after $1 \mathrm{~min}$ with a microplate reader (Synergy HTX; BioTek Instruments Inc.). Finally, the GPx activity in the salivary samples was reported in $\mathrm{U} / \mathrm{mL}$ as $M \pm S D$.

\section{Statistical analysis}

Data was expressed as $M \pm S D$. Statistical analysis was performed using the SPSS for Windows software, v. 16.0 (SPSS Inc., Chicago, USA). For statistical analysis, Spearman's correlation test, the Mann-Whitney $U$ test and the Kruskal-Wallis test were used with the software. Statistical significance was set at $p<0.05$.

\section{Results}

In this cross-sectional study, 90 children aged 7-12 years participated. Thirty-six (40\%) of them were girls and 54 (60\%) were boys. Since the children had mixed dentition, the average caries index was calculated based on the sum of the decayed, missing, filled teeth in permanent/primary dentition (DMFT/dmft). This index was $7.17 \pm 3.56$ in our study. No significant correlation was established between the SOD, CAT and GPx activity and DMFT/dmft $(p>0.05)$ (Fig. 1A,1C,1E). 

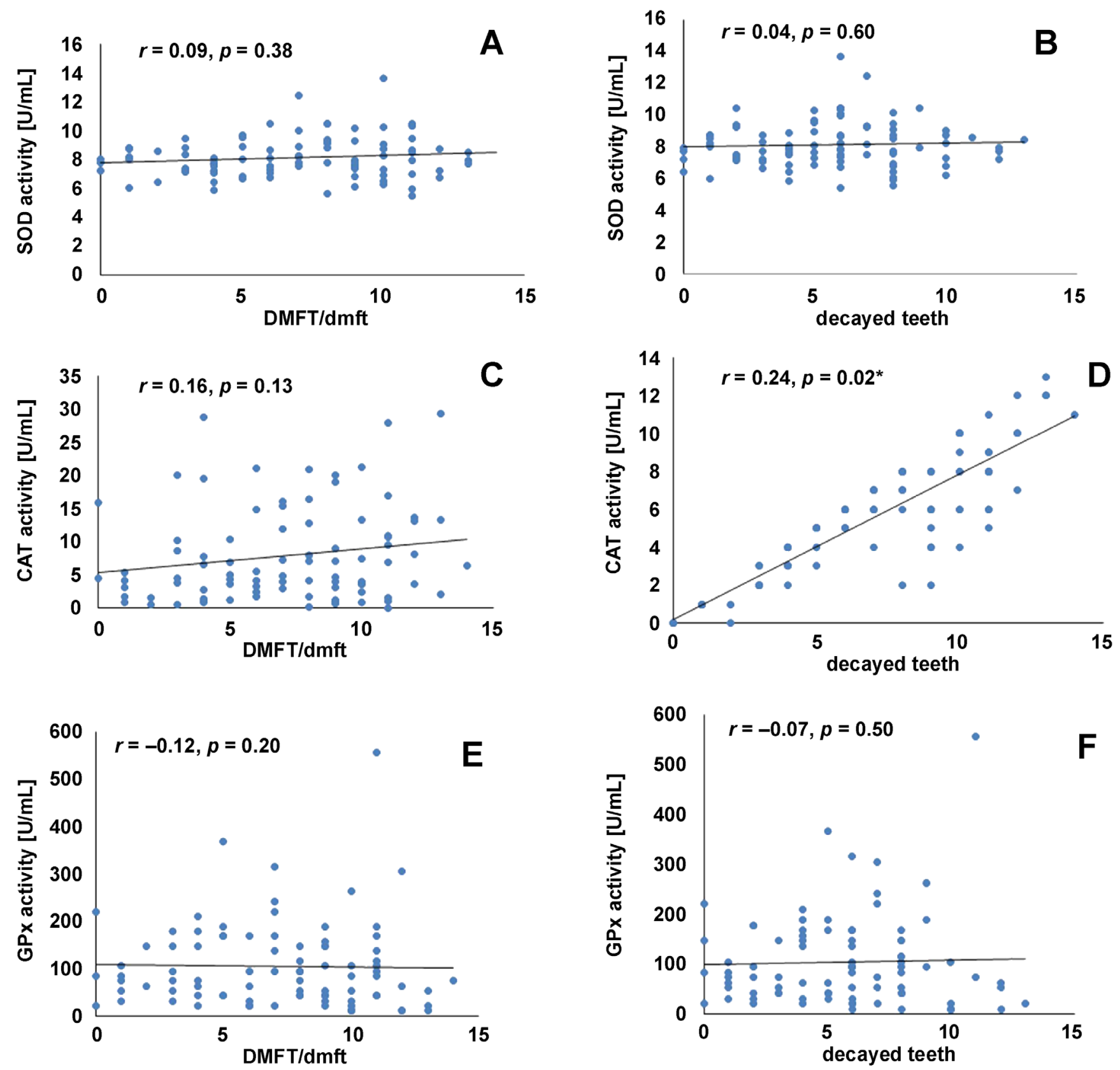

Fig. 1. Associations between salivary enzymatic antioxidants and the decayed, missing, filled teeth in permanent/primary dentition index (DMFT/dmft) as well as the decayed teeth

A - SOD activity and DMFT/dmft; B - SOD activity and the decayed teeth; C - CAT activity and DMFT/dmft; D - CAT activity and the decayed teeth; E - GPx activity and DMFT/dmft; F- GPx activity and the decayed teeth.

SOD - superoxide dismutase; CAT - catalase; GPX - glutathione peroxidase; * statistical significance ( $p<0.05$; Spearman's correlation test).

The CAT activity showed a positive correlation with the total number of decayed teeth $(r=0.24 ; p=0.02)$ (Fig. 1D), but the SOD and GPx activity showed no correlation $(r=0.04 ; p=0.60$ and $r=-0.07 ; p=0.50$, respectively) (Fig. 1B,1F).

Spearman's correlation test showed a positive correlation between the SOD activity and the frequency of tooth brushing; the positive direction of the relationship indicates that the SOD activity increases with increased tooth brushing frequency $(p=0.03)$ (Fig. 2A). The CAT and GPx activity was not correlated with the frequency of tooth brushing ( $p>0.05$ ) (Fig. 2B,2C).The Mann-Whitney $U$ test showed no significant differences in the activity of enzymatic antioxidants between genders $(p>0.05)$. The test showed higher values of the SOD, CAT and GPx activity in boys in comparison with girls, but the differences were statistically nonsignificant (Table 1). As shown in Fig. 3A-C, there was an inverse relationship between the SOD $(r=-0.04 ; p<0.001)$, CAT $(r=-0.07 ; p<0.001)$ and GPx $(r=-0.08, p<0.001)$ activity and age. 

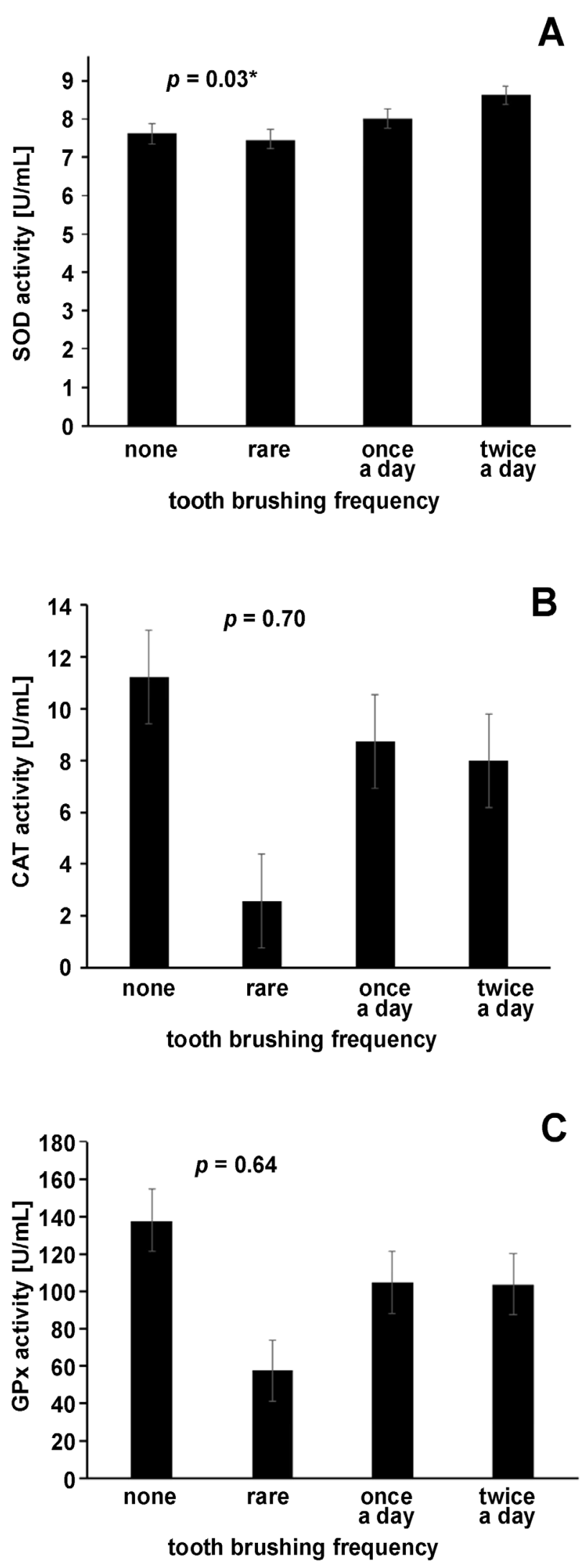

Fig. 2. Correlation between salivary enzymatic antioxidants and tooth brushing frequency

A - salivary SOD activity and tooth brushing frequency; B - salivary CAT activity and tooth brushing frequency; C - salivary GPx activity and tooth brushing frequency.

* statistical significance ( $p<0.05$; Spearman's correlation test).
Table 1. Comparison of the activity of the salivary antioxidants between the genders (Mann-Whitney $U$ test)

\begin{tabular}{|l|c|c|c|}
\hline \multicolumn{1}{|c|}{ Antioxidant } & Male & Female & $p$-value \\
\hline SOD $[\mathrm{U} / \mathrm{mL}]$ & $8.14 \pm 1.49$ & $8.05 \pm 1.38$ & 0.641 \\
CAT $[\mathrm{U} / \mathrm{mL}]$ & $8.39 \pm 7.26$ & $7.06 \pm 6.89$ & 0.447 \\
GPx $[\mathrm{U} / \mathrm{mL}]$ & $112.42 \pm 103.37$ & $90.08 \pm 65.43$ & 0.516 \\
\hline
\end{tabular}

Data presented as mean $(M) \pm$ standard deviation (SD).
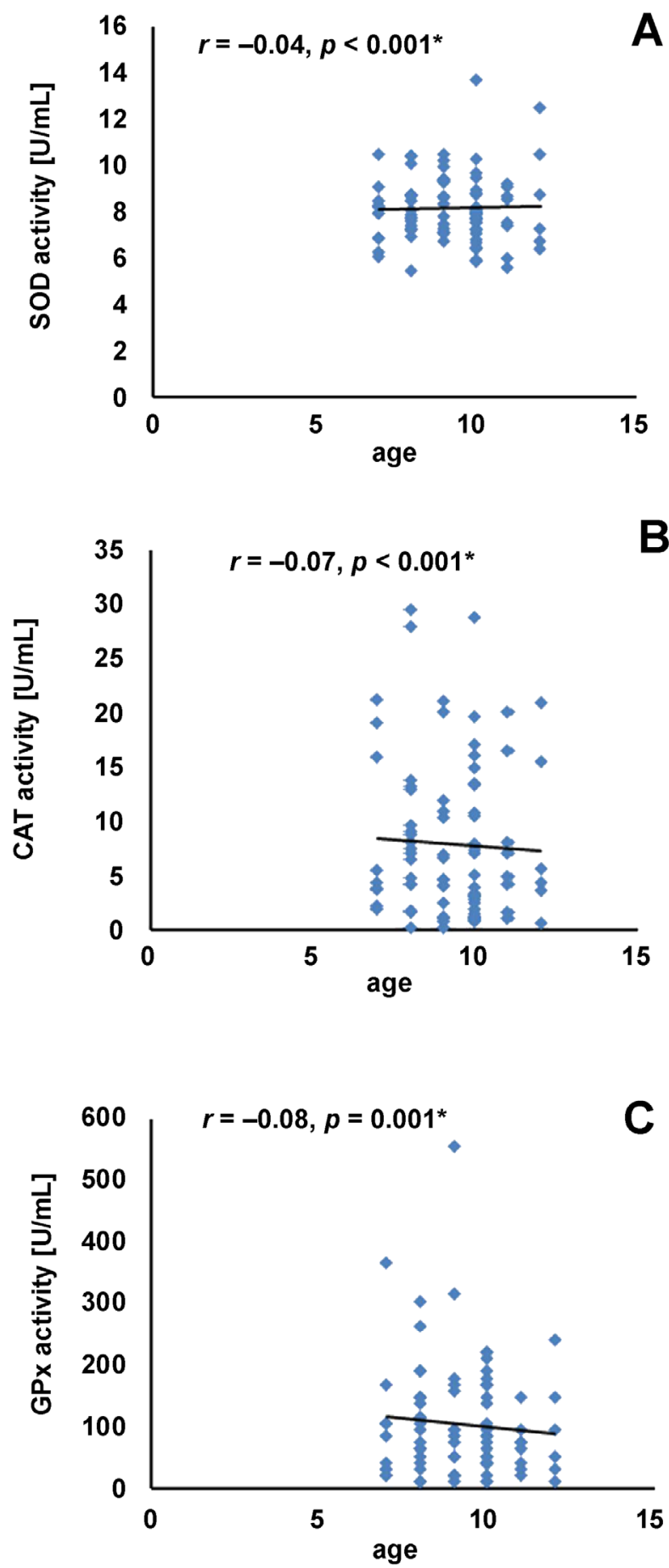

Fig. 3. Association between salivary enzymatic antioxidants and age A - SOD activity and age; B - CAT activity and age; C - GPx activity and age. * statistical significance ( $p<0.001$; Spearman's correlation test). 


\section{Discussion}

In the present study, there was no significant relationship between the SOD activity and DMFT/dmft. Hendi et al. reported a decrease in the SOD activity in children with caries as compared to children without caries. ${ }^{4}$ In contrast, some studies showed that the salivary SOD activity in children with severe early childhood caries (S-ECC) is higher than in children without caries. ${ }^{2,6}$ Silva et al. showed that the salivary SOD activity in children with S-ECC was significantly higher than in children without caries. ${ }^{6}$ Hedge et al. reported a significant increase in the salivary SOD activity in the group with DMFT $>10$ as compared to DMFT $=0 .^{2}$ The most important reason for the differences between our results and the abovementioned studies might be the sampling methods and techniques used. In those case-control studies, the enzymatic activity was compared between 2 groups with great DMFT differences. This may explain their significant results. ${ }^{2,6}$ In contrast, in our cross-sectional study, children with varying degrees of dental caries were not divided into different groups due to the small sample size of the caries-free group.

In the present study, a significant and positive association was observed between the CAT activity and the number of decayed teeth, like in the study done by Hendi et al. ${ }^{4}$

Our results showed no significant relationship between the salivary GPx activity and DMFT/dmft. This finding is consistent with the results of Hedge et al. ${ }^{11}$ They also observed no significant difference in the GPx activity of subgroups with different DMFT/dmft (DMFT < 3; $3<$ DMFT < 10; and DMFT > 10). However, they showed a significant increase in the salivary GPx activity in the group with active caries as compared to the caries-free group.11 In our study, such a comparison was not made due to the small number of caries-free subjects.

Previous studies reported a significant elevation of the salivary total antioxidant capacity (TAC) in patients with an advanced form of caries (cavitated lesions) in comparison with a non-caries group. ${ }^{2,8,19,20}$ Furthermore, the elevation of TAC in children with a high caries rate may be due to the colonization of microorganisms as well as the stimulation of the immune system, which consequently results in the body's compensatory reaction to reduce oxidative stress. ${ }^{6}$ Tóthová et al. showed that an improvement in the clinical parameters of the Oral Hygiene Index $(\mathrm{OHI})$ reduced the concentrations of thiobarbituric acid reacting substances (TBARS) as markers for increasing TAC. $^{3}$ In the present study, the SOD activity increased with the increased frequency of tooth brushing whereas there was no relationship between the CAT and GPx activity and the tooth brushing frequency.

Several studies have also shown a positive relationship between periodontal parameters and enzymatic antioxidants. Novaković et al. showed increased SOD and GPX activity in patients with chronic periodontitis after non-surgical treatment. ${ }^{21}$ An in vitro study also showed that the SOD and CAT activity diminished with the increasing depth of the sulcus in periodontal patients. ${ }^{22}$ A medication with liposome-encapsulated SOD improves the healing process by reducing periodontal inflammatory reactions. However, such an effect was not observed in the case of liposome-encapsulated CAT. $^{22}$

Our study showed there was a reduction in the activity of SOD, CAT and GPx with age. This relationship is weaker for SOD in comparison with CAT and GPx. To support this finding, there are studies that show a positive relationship between age and TAC. For example, Tóthová et al. ${ }^{16}$ and Hegde et al. ${ }^{23}$ showed the elevation of TAC with age in 4-18-year-old children and in children aged $<71$ months as well as in 6-12-year-olds, respectively. In contrast to the abovementioned studies, Kumar et al. showed no significant relationship between the children's age (age range: $3-5$ years) and TAC. ${ }^{19}$

In our study, the activity of SOD, CAT and GPx was not significantly different between genders. In contrast to this finding, the results of Ahmadi-Motamayel et al. showed higher TAC in boys than in girls in the age range of 15-17 years. ${ }^{24}$ Moreover, Hendi et al. reported lower CAT activity and higher GPx activity in the female group as compared to the male group. ${ }^{4}$ Since the performance of the antioxidant system generally depends on various factors, including TAC, the amount of the released free radicals, genetics, diet, age, hormones as well as individual stress, ${ }^{25}$ differences in the results of various studies may be attributed to differences in those factors. ${ }^{23}$ Finally, more clinical and laboratory studies are also needed to clarify the complete relationship between enzymatic antioxidants and dental caries.

\section{Conclusions}

In conclusion, since few studies have been carried out on enzymatic antioxidants and dental caries, the present study has provided new insights about the activity of enzymatic antioxidants in children with varying degrees of dental caries. The CAT activity increased with an increase in the number of decayed teeth. The SOD activity was positively correlated with the tooth brushing frequency. There was a decrease in the SOD, CAT and GPx activity with advancing age. The activity of SOD, CAT and GPx activity was higher in boys than in girls.

Since the frequency of tooth brushing was evaluated as one of the indicators of oral health, further studies are needed to determine the exact relationship between the salivary enzymatic antioxidant activity and the oral hygiene score. Also, further extensive studies with larger sample sizes are required, which might lead to some significant results. Moreover, case-control studies are suggested in the future. 


\section{ORCID iDs}

Zakaria Vahabzadeh (i) https://orcid.org/0000-0002-9854-9653 Zahra Moaven Hashemi (1) https://orcid.org/0000-0001-5711-9094 Bijan Nouri (D) https://orcid.org/0000-0002-2932-5058 Fatemeh Zamani (D) https://orcid.org/0000-0002-0795-8276

Faranak Shafiee (D) https://orcid.org/0000-0002-7280-2235

\section{References}

1. Featherstone JDB. Dental caries: A dynamic disease process. Aust Dent J. 2008;53(3):286-291. doi:10.1111/j.1834-7819.2008.00064.x

2. Hegde MN, Hegde ND, Ashok A, Shetty S. Biochemical indicators of dental caries in saliva: An in vivo study. Caries Res. 2014;48(2):170-173. doi:10.1159/000355580

3. Tóthová L, Kamodyová N, Červenka T, Celec P. Salivary markers of oxidative stress in oral diseases. Front Cell Infect Microbiol. 2015:5:73. doi:10.3389/fcimb.2015.00073

4. Hendi SS, Goodarzi MT, Moghimbeigi A, Ahmadi-Motamayel F. Evaluation of the status of salivary antioxidants in dental caries. Infect Disord Drug Targets. 2019. doi:10.2174/1871526519666191031100432

5. Hegde MN, Attavar SH, Shetty N, Hegde ND, Hegde NN. Saliva as a biomarker for dental caries: A systematic review. J Conserv Dent. 2019;22(1):2-6. doi:10.4103/JCD.JCD_531_18

6. Silva PVD, Troiano JA, Nakamune ACMS, Pessan JP, Antoniali C. Increased activity of the antioxidants systems modulate the oxidative stress in saliva of toddlers with early childhood caries. Arch Oral Biol. 2016;70:62-66. doi:10.1016/j.archoralbio.2016.06.003

7. Hegde MN, Hegde ND, Ashok A, Shetty S. Evaluation of total antioxidant capacity of saliva and serum in caries-free and cariesactive adults: An in-vivo study. Indian J Dent Res. 2013;24(2):164-167. doi:10.4103/0970-9290.116670

8. Jurczak A, Kościelniak D, Skalniak A, Papież M, Vyhouskaya $P_{\text {, }}$ Krzyściak $W$. The role of the saliva antioxidant barrier to reactive oxygen species with regard to caries development. Redox Rep. 2017;22(6):524-533. doi:10.1080/13510002.2017.1301625

9. Lopes Castro MM, Duarte NN, Nascimento PC, et al. Antioxidants as adjuvants in periodontitis treatment: A systematic review and meta-analysis. Oxid Med Cell Longev. 2019;2019:9187978. doi:10.1155/2019/9187978

10. Ighodaro OM, Akinloye OA. First line defence antioxidants - superoxide dismutase (SOD), catalase (CAT) and glutathione peroxidase (GPX): Their fundamental role in the entire antioxidant defence grid. Alexandria J Med. 2018;54(4):287-293. doi:10.1016/j.ajme.2017.09.001

11. Hegde MN, Kumari S, Hegde ND, Shetty SS. Myeloperoxidase and glutathione peroxidase activity of saliva and serum in adults with dental caries: A comparative study. J Free Radic Antioxid Photon. 2013;139(2):175-180. https://www.researchgate.net/publication/236622132_Myeloperoxidase_and_Glutathione_Peroxidase_Activity_of_Saliva_and_Serum_in_Adults_with_Dental_Caries_A_Comparative_Study. Accessed July 1, 2020.

12. Góth $L$, Rass $P$, Páy $A$. Catalase enzyme mutations and their association with diseases. Mol Diag. 2004;8(3):141-149. doi:10.1007/BF03260057

13. Karincaoglu Y, Batcioglu K, Erdem T, Esrefoglu M, Genc M. The levels of plasma and salivary antioxidants in the patient with recurrent aphthous stomatitis. J Oral Pathol Med. 2005;34(1):7-12. doi:10.1111/ j.1600-0714.2004.00253.x

14. Weydert CJ, Cullen JJ. Measurement of superoxide dismutase, catalase and glutathione peroxidase in cultured cells and tissue. Nat Protoc. 2010;5(1):51-66. doi:10.1038/nprot.2009.197

15. Livnat G, Bentur L, Kuzmisnsky E, Nagler RM. Salivary profile and oxidative stress in children and adolescents with cystic fibrosis. J Oral Pathol Med. 2010;39(1):16-21. doi:10.1111/j.1600-0714.2009.00813.x

16. Tóthová L, Celecová V, Celec P. Salivary markers of oxidative stress and their relation to periodontal and dental status in children. Dis Markers. 2013;34(1):9-15. doi:10.3233/DMA-2012-00943

17. Dean J, ed. McDonald and Avery's Dentistry for the Child and Adolescent. $10^{\text {th }}$ ed. St. Louis, MO: Mosby (Elsevier Health Sciences); 2016:135.

18. Yamuna Priya K, Muthu Prathibha K. Methods of collection of saliva - a review. Int J Oral Health Dent. 2017;3(3):149-153. doi:10.18231/2395-499X.2017.0032
19. Kumar D, Pandey RK, Agrawal D, Agrawal D. An estimation and evaluation of total antioxidant capacity of saliva in children with severe early childhood caries. Int J Paediatr Dent. 2011;21(6):459-464. doi:10.1111/j.1365-263X.2011.01154.x

20. Preethi $B P$, Reshma $D$, Anand $P$. Evaluation of flow rate, $\mathrm{pH}$, buffering capacity, calcium, total proteins and total antioxidant capacity levels of saliva in caries free and caries active children: An in vivo study. Indian J Clin Biochem. 2010;25(4):425-428. doi:10.1007/s12291-010-0062-6

21. Novaković N, Cakić S, Todorović T, et al. Antioxidative status of saliva before and after non-surgical periodontal treatment. Srp Arh Celok Lek. 2013;141(3-4):163-168. doi:10.2298/sarh1304163n

22. Trivedi S, Lal N. Antioxidant enzymes in periodontitis. J Oral Biol Craniofac Res. 2017;7(1):54-57. doi:10.1016/j.jobcr.2016.08.001

23. Hegde AM, Rai K, Padmanabhan V. Total antioxidant capacity of saliva and its relation with early childhood caries and rampant caries. J Clin Pediatr Dent. 2009;33(3):231-234. doi:10.17796/jcpd.33.3.c730518021m56077

24. Ahmadi-Motamayel F, Goodarzi MT, Hendi SS, Kasraei S, Moghimbeigi A. Total antioxidant capacity of saliva and dental caries. Med Oral Patol Oral Cir Bucal. 2013;18(4):e553-e556. doi:10.4317/medoral.18762

25. Pereslegina IA. The activity of antioxidant enzymes in the saliva of normal children [in Russian]. Lab Delo. 1989;11(1):20-23. PMID:2481094 